\title{
Determination of Equilibrium Points for Steady State Conservative Models
}

\author{
Tatiana Panikovskaya, Ural Federal University named after the first President of Russia B.N. Yeltsin
}

\begin{abstract}
The development and a more sophisticated structure of electric power systems, the transition towards deregulation and competition between the participants of the electric energy market cause the necessity of a complex steady state analysis, the evaluation of the electric power system stability, with its parameters being changed. The paper addresses the steady state analysis based on algebraic methods. Regular (trivial) and non-regular (additional) equilibrium points for the electric power system conservative model are determined. Changing regular and non-regular equilibrium points in a heavily loaded electric power system is studied.
\end{abstract}

Keywords - conservative model, electric power system, steady state, regular equilibrium point, non-regular equilibrium point, algebraic solution.

\section{INTRODUCTION}

Calculations of the steady state conditions are among the problems most often solved in designing and operating electric power systems (EPS). They are aimed at identifying the main regime parameters, which depends upon the way the initial information is set up and the physical content of the process under consideration.

For the electric power system the model of which is represented by the combination of the synchronous machines rotor movements, the problem of the steady state regime calculation is confined to determining vector $\delta^{e}$ of the equilibrium states. With the solutions being analyzed, the socalled "group properties" are specified, that appear in varying the parameters of the power system elements.

\section{PROBLEM DESCRIPTION}

For the conservative model made the assumption of a purely reactive nature of the electrical connections between the generators. The electric power system will be given by following algebraic equations:

$$
\sum_{j=0}^{n} q_{i j} \sin \left(\delta_{i}-\delta_{j}\right)=p_{i}, i=\overline{1, n}
$$

$q_{i j}$ - are matrix elements of the generators' mutual capacities; $\delta_{i j}$ - are the voltage angle.

The solutions of equations system (1) can be found using direct methods, they will correspond to the equilibrium point $\delta^{e}$. To study steady state regimes solutions, the methods which suggest confining the system of transcendental equations (1) to algebraic equations system are of special interest. The key idea of it is to avoid singularity of power flow Jacobean caused by load of buses reaching the critical condition or bifurcation point.

Determining the equilibrium points (EP) for a power system comprising three generators is described in [1]. In the conservative model with $n=2$ direct determining EP is impossible so a method for solving equations in (1) relative to a new variable $x_{i}=\sin \delta_{i}$ by reducing it to an algebraic equation of the sixth order $\sum_{k=0}^{6} a_{k i} x_{i}^{6-k}=0$, where factors $a=f(P, Q)$ are functions of parameters $y_{i j}, p_{i}$.

For the conservative model with $n \geq 3$ system (1) can be transformed into the system of the algebraic equations $(n-1)$ of s degree relative to variable $x_{i} \equiv \sin \delta_{i}, y_{j} \equiv \operatorname{tg}\left(\delta_{j} / 2\right)$, $z_{k} \equiv \operatorname{tg}\left(\delta_{k} / 4\right), i \neq j \neq k$ by eliminating variable $x_{i}$ and subsequent changes:

- Irrationality eliminations in the equations (1);

- Introduction of new variables $y_{j} \equiv \operatorname{tg}\left(\delta_{j} / 2\right)$, $z_{k} \equiv \operatorname{tg}\left(\delta_{k} / 4\right), i \neq j \neq k$.

In a new system of the algebraic equations, variable $x_{i}=\sin \delta_{i}$ is of the most advanced degree. The degree size $x_{i}$ depends on the number of generators and is equal to $3 \cdot 2^{n-1}$.

The system consisting of $m$ algebraic equations with $n$ unknowns can be analyzed by the method described in [2]. Its solutions are confined to the a solution of an equation with one unknown and the solution of a system of equations $m-1$ with $n-1$ of unknowns. The latter, in turn, is reduced to a system with still a smaller number of equations and unknowns. Ultimately, the analysis of an arbitrary system of equations is reduced to the solution of several equations, each of which has only one unknown.

The system of equations is of the following form:

$$
\left.\begin{array}{l}
H_{1}\left(x_{2}, x_{3}, \ldots, x_{n}\right) x_{1}^{k}+G_{1}\left(x_{1}, x_{2}, \ldots, x_{n}\right)=0 \\
H_{2}\left(x_{2}, x_{3}, \ldots, x_{n}\right) x_{1}^{\ell}+G_{2}\left(x_{1}, x_{2}, \ldots, x_{n}\right)=0
\end{array}\right\}
$$

where $0<k \leq l ; \quad H_{1}\left(x_{2}, x_{3}, \ldots, x_{n}\right), H_{2}\left(x_{2}, x_{3}, \ldots, x_{n}\right)$, $G_{1}\left(x_{1}, x_{2}, \ldots, x_{n}\right), G_{2}\left(x_{1}, x_{2}, \ldots, x_{n}\right)$ are polynomials, degree $G_{1}\left(x_{1}, x_{2}, \ldots, x_{n}\right)$ is less than $k$, and degree 
$G_{2}\left(x_{1}, x_{2}, \ldots, x_{n}\right)$ is less than $\ell$.

The equation in (2) can be transformed into two new systems:

$$
\begin{aligned}
& H_{1}\left(x_{2}, x_{3}, \ldots, x_{n}\right)=0 \\
& G_{1}\left(x_{1}, x_{2}, \ldots, x_{n}\right)=0 \text {. } \\
& H_{1}\left(x_{2}, x_{3}, \ldots, x_{n}\right) H_{2}\left(x_{2}, x_{3}, \ldots, x_{n}\right) x_{1}^{\ell}+ \\
& +H_{1}\left(x_{2}, x_{3}, \ldots, x_{n}\right) G_{2}\left(x_{1}, x_{2}, \ldots, x_{n}\right)=0 .
\end{aligned}
$$

Equation (4) is transformed to a form:

$$
\begin{aligned}
& H_{1}\left(x_{2}, x_{3}, \ldots, x_{n}\right) G_{2}\left(x_{1}, x_{2}, \ldots, x_{n}\right)- \\
& \quad-H_{2}\left(x_{2}, x_{3}, \ldots, x_{n}\right) G_{1}\left(x_{1}, x_{2}, \ldots, x_{n}\right) x_{\ell}^{\ell-k}=0 .
\end{aligned}
$$

Transforming a system of transcendental equations (1) into an algebraic equations system is carried out in the following way. The variable $x_{i} \equiv \sin \delta_{i}$ has been expressed through functions $\sin \delta_{j}, \cos \delta_{j} \quad$ and $\sin \delta_{k}, \cos \delta_{k}$ $i \neq j \neq k ; i, j, k \in(1,3)$. Functions $\sin \delta_{j}, \cos \delta_{j}$ were sequentially substituted by $y_{j} \equiv \operatorname{tg}\left(\delta_{j} / 2\right), \quad\left|\delta_{j}\right|<\pi$; $\sin \delta_{k}, \cos \delta_{k}-z_{k} \equiv \operatorname{tg}\left(\delta_{k} / 4\right),\left|\delta_{k}\right|<\pi$. As a result, an identical set of equations is obtained:

$$
\left.\begin{array}{l}
\sum_{l=0}^{6} b_{l}\left(z_{k}\right) y_{j}^{6-k}=0 \\
\sum_{l=0}^{6} c_{l}\left(z_{k}\right) y_{j}^{4-k}=0
\end{array}\right\}
$$

The solution for $n=3$ can be determined in the following way: the factor elements values $B\left(z_{k}\right), C\left(z_{k}\right)$ are determined, then by consecutive step-by-step change $\delta_{k}$ from $-\pi$ to $\pi$ points $\delta_{k}$ where the system (6) resultant is equal to zero are defined. Then, for the determined angle $\delta_{k}$ corresponding angles $\delta_{i}, \delta_{j}$ are calculated.

Imagine a system of the equations (1) as dependent upon some parameters $t_{1}, t_{2}$ :

$$
\sum_{j=0}^{n}\left(q_{\min }+t_{1}\left(q_{i j}-q_{\min }\right)\right) \cdot \sin \left(\delta_{i}-\delta_{j)}=t_{2} p_{i}, i=\overline{1, n}\right.
$$

The variable $q_{\min }$ is the minimum value out of $q_{i j}, i \neq j, i, j \in(0, n) ; t_{1}, t_{2}$ are independent parameters ranging from 0 to 1 .

Expression (7) at $t_{1}, t_{2}=0$ is termed a generating system with two known types of equilibrium positions (EP): trivial (zero) and additional (nonzero) [3].

Trivial equilibrium points are regular positions of balance where partial interactions of each generation are equal to zero. The analysis of trivial EP is implemented, the number of which for the system with $n$ degrees of freedom is equal to $2^{n}$, the number of additional EP does not exceed $n$ !. Additional equilibrium positions belong to the boundary space planes $\Delta_{n}^{s}\left\{\delta_{1}, \delta_{2}, \ldots, \delta_{n}\right\}$, partial synchronous machines' interactions for them may be other than zero $\left(\delta_{i j}^{e p}=\delta_{i}^{e p}-\delta_{j}^{e p}\right.$ is not always equal to $0(\pi)$ ).

With system parameters (7) randomly varied, additional equilibrium points will move along some trajectories defined in $n$ - measured space $\Delta_{n}^{\pi}\left\{\delta_{1}, \delta_{2}, \ldots, \delta_{n}\right\}$. These trajectories originate from the determined irregular equilibrium points of a generating system. Changing additional EP under the conditions of $q_{i 0}=q_{j 0}=q_{k 0}, \quad q_{i j}=q_{i k}=q_{j k}=q_{\min }$, $i \neq j \neq k ; i, j, \quad i, j, k \in(1, n)$ and changing $t_{1}$ from 1 to 0 are considered below.

In the generating system, for add $n$, all the positions of equilibrium having the maximal meaning (importance) of potential energy are degenerate (Hessian of the potential energy is equal to zero $H(\delta)=0)$. Regular $(\pi, 0, \pi)$, $(\pi, \pi, 0),(0, \pi, \pi)$ and irregular combinations presented as $\left( \pm \frac{\pi}{2}, \mp \frac{\pi}{2}, \pi\right), \quad\left(\pi, \pm \frac{\pi}{2}, \mp \frac{\pi}{2}\right), \quad\left( \pm \frac{\pi}{2}, \pi, \mp \frac{\pi}{2}\right) \quad$ for $\delta_{0}=0$ belong to these equilibrium positions. Solving system (7) allows to determine the general properties inherent to the changes of solutions of the steady state equations when the parameters of electric power system are being varied.

Degenerate discrete EP with any odd $n$ lines are connected by lines $h^{n}\left(\delta_{1}, \delta_{2}, \ldots, \delta_{n}\right)$ and constitutes a continuous set. When moving along such lines the change of potential energy does not occur in $\Delta_{n}^{\pi}$. Consequently, these lines are the edges of the equipotential surface.

Let's analyze the solution of system (7) with changing $q_{i j}$ and $t_{1}=1$ for the conservative model of a power system. The system (7) has double zero roots:

$$
y_{j}^{2}=0, z_{k}^{2}=0
$$

and a great many valid solutions which are equation solutions:

$$
\begin{aligned}
& \left\{\left(q_{i 0}^{2}-q_{i j}^{2}\right)+\left(q_{i 0}^{2}+3 q_{i j}^{2}-4 q_{i 0} q_{i j}\right) z_{k}^{2}\right\} y_{j}^{2}+ \\
& +8 q_{i j} z_{k} y_{j}+\left(q_{i 0}^{2}-q_{i j}^{2}\right) z_{k}^{2}+\left(q_{i 0}^{2}+3 q_{i j}^{2}-4 q_{i 0} q_{i j}\right)=0 .
\end{aligned}
$$

A certain curve characterized by the property that the coordinates of any point $A\left(y_{j a}, z_{k a}\right)$ on this curve satisfy this equation corresponds to equation (9). For any value of 
variable $z_{k a}$ a fixed value the variable $y_{i a}$ can be determined (and vice versa).

The analysis of system (8) solutions shows that regular EP do not changes their position with the parameters of the system under investigation being changed. The irregular EP may disappear, keep your contacts information (preserve their coordinates) or change them and move into non-degenerated equilibrium positions when varying values $q_{i j}$. Changing irregular equilibrium points is determined by the analysis of solutions (9).

\section{RESEARCH RESULTS}

For the equation (9) to have real solutions, it is necessary and sufficient to observe the condition of:

$$
q_{i 0}^{2}+3 q_{i j}^{2}-4 q_{i 0} q_{i j} \leq 0, i \neq j ; i, j \in(1,3)
$$

The equation (10) is transformed into:

$$
\left(q_{i 0} / q_{i j}\right)^{2}-4\left(q_{i 0} / q_{i j}\right)+3 \leq 0
$$

And allows receiving the following condition $1 \leq\left(q_{i 0} / q_{i j}\right) \leq 3$.

Thus, the solutions of the equations system (9) will exist if $\left(q_{i 0} / q_{i j}\right) \in[1,3]$, with the solution of the equation (9) at $\left(q_{i 0} / q_{i j}\right)=3$ coinciding with the solution of system (6).

The system of equations (1) allows the solution to be determined, which corresponds to the chosen EPS parameters and for $\operatorname{tg}\left(\delta_{j} / 2\right)=r \cdot \operatorname{tg}\left(\delta_{k} / 2\right) \quad(r$ is the factor which can be both negative and positive [4])

The set zero degenerated EP for a generating system represents a closed polygon (Fig. 1) the parties (sides) of which are straight lines $h^{n}\left(\delta_{1}, \delta_{2}, \delta_{3}\right)=j$. The equations of straight lines are defined as:

$$
\delta_{i}= \pm \pi, \operatorname{tg} \frac{\delta_{j}}{2} \cdot \operatorname{tg} \frac{\delta_{k}}{2}=-1, i \neq j \neq k ; i, j, k \in(1,3)
$$

With $q_{i 4}>1$, the continuous set of degenerated equilibrium positions is disintegrated into discrete sets, the straight lines $h^{n}=0$ turn to curves and are determined from following relations:

$$
\left.\begin{array}{l}
\delta_{i}=\arcsin \left(-\left(\sin \delta_{j}+\sin \delta_{k}\right)\right), \\
2 q_{i 0}^{2}+2 q_{i j}^{2}+4 q_{i 0} q_{i j}+ \\
+\left(q_{i 0}^{2}+3 q_{i j}^{2}-4 q_{i 0} q_{i j}\right) \operatorname{tg}^{2} \frac{\delta_{j}}{2} \operatorname{tg}^{2} \frac{\delta_{k}}{2}+ \\
+8 q_{i j} \operatorname{tg} \frac{\delta_{j}}{2} \operatorname{tg} \frac{\delta_{k}}{2}+\left(q_{i 0}^{2}-q_{i j}^{2}\right) / \operatorname{tg}^{2} \frac{\delta_{k}}{2}=0 .
\end{array}\right\}
$$

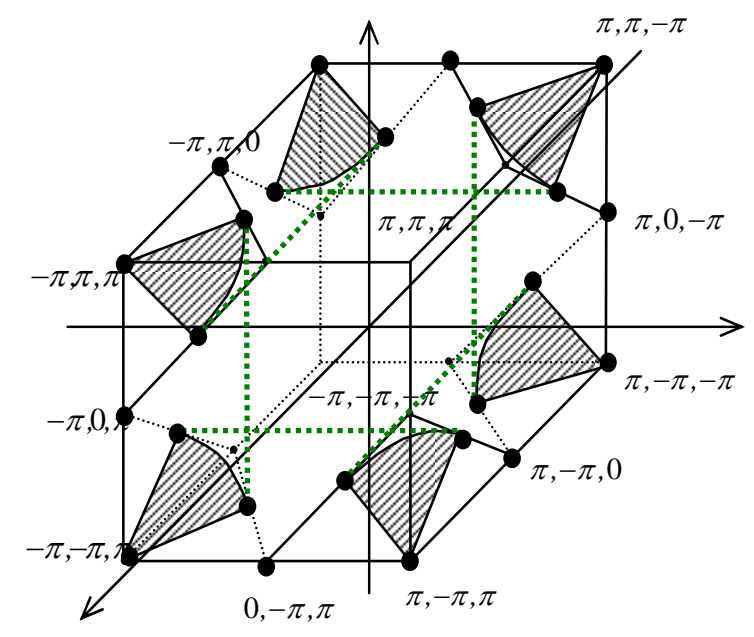

Fig. 1. A set of degenerated equilibrium points for the generating system

Fig. 2 shows the discrete sets corresponding to a degenerated equilibrium positions for a system with $q_{i 0}=q_{j 0}=q_{k 0}=2, q_{i j}=q_{i k}=q_{j k}=1$. A dot-and- dashed line shows a change of one of variables $\delta_{i}$ by $2 \pi$.

Curves $h^{n}=0$ belong to the surface $H^{n}=0$ covering area of minimums of potential energy, are closed relative to the origin of coordinates and possess the following properties:

1. If $q_{i 0}=q_{i j}=1$ curves $h^{n}=0$ represent a closed and symmetric relative to the origin of coordinates polygon consisting of pieces of straight lines determined by (12).

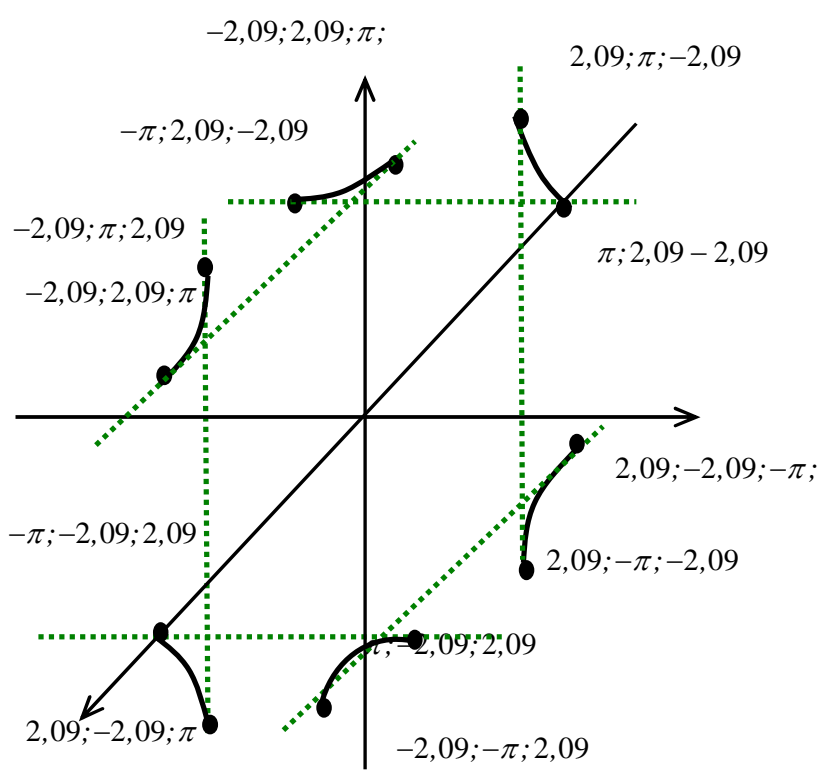

Fig. 2. The discrete sets of degenerated equilibrium points 
2. At $1<q_{i 0} / q_{i j}<3$ curves cross the planes $h^{n}=0$ in points $\delta_{i}= \pm \pi$ which are solutions of the equation (9) in case $\operatorname{tg} \frac{\delta_{j}}{2}=\operatorname{tg} \frac{\delta_{k}}{2}$ or $\delta_{j}=-\delta_{k}$, and represent pieces of curves in the space limited by planes $\delta_{i}= \pm \pi$.

3. Curves $h^{n}=0$ turn into points with coordinates $\delta_{i}= \pm \pi, \delta_{j}=-\delta_{k}=\pi$ if $q_{i 0} / q_{i j}=3$. At these points, irregular equilibrium points merge with the regular ones. The further expansion in the relation $q_{i 0} / q_{i j}$ causes irregular EP to disappear. Consequently, with the electric rigidity of system increasing, curves $h^{n}=0$ converge and disappear at points of $(\pi, \pi,-\pi)$ type. The direction of the curves position change in the space $\Delta_{n}^{\pi}$ is shown by arrows in Fig. 3 .

4. When the designation of the component is changed, the equations system (6) form does not undergo any essential changes; only values of factors $B\left(z_{k}\right), C\left(z_{k}\right)$ change. It means that the curves $h^{n}=0$ are, but remain symmetric relative to the origin of coordinates.

\section{CONCLUSION}

Thus, the system of the equations of the electric power system steady state can generally be confined to systems of the algebraic equations which can be solved using conventional methods. The irregular equilibrium points of electric power systems unloaded conservative models can be represented by some invariant sets.

Results will be used for qualitative analysis of stationary regimes of power systems with distributed generation. Next step, we will do more work in this direction.

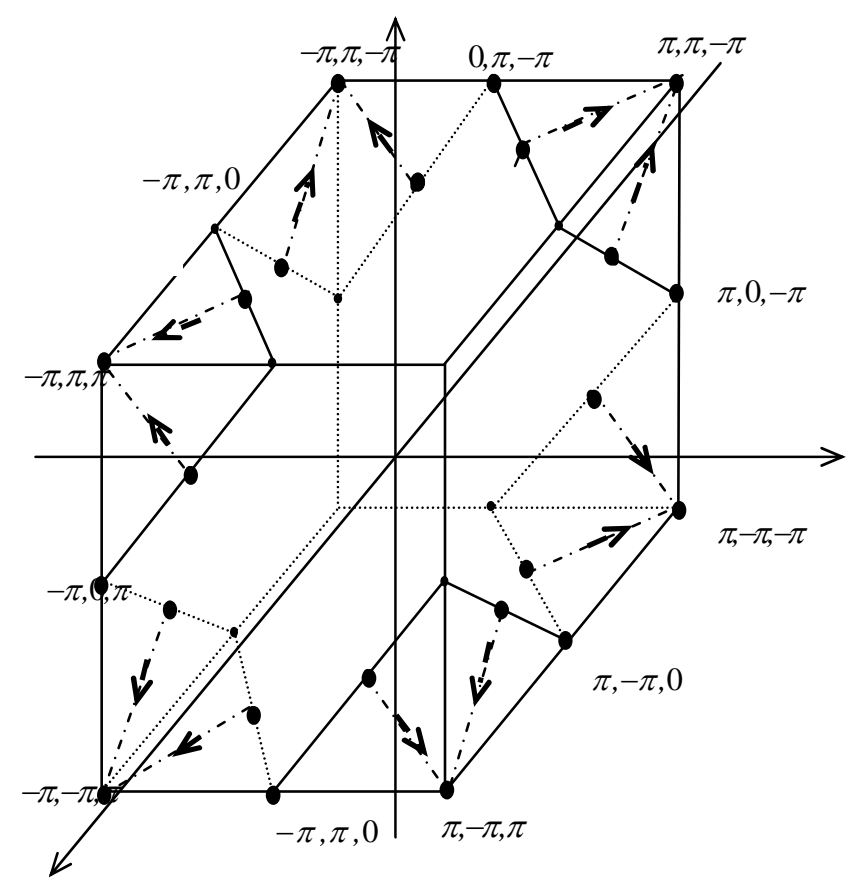

Fig.3. Change the position of curves $h^{n}=0$ in space $\Delta_{n}^{\pi}$

\section{ACKNOWLEDGMENT}

The author expresses his gratitude to his mentor Mstislav Petrovich Rudnitsky.

\section{REFERENCES}

[1] Rudnitsky M.P., "Element of the theory of stability and management of modes of electrical power systems," Sverdlovsk: UPI, 1984.

[2] Mishina A.P., Proskurykov I.V., "Hidger algebra: linear algebra, polynomial, general algebra," M.: Fizmatgiz, 1962.

[3] Panikovskaya T.Y., Rudnitsky M.P., "Irregular positions of equilibrium of conservative models of electric power systems," Conference "The raise of a production efficiency and use of an energy in conditions of Siberia," Irkutsk, 1990.

[4] Kiseleva I.P., Pavlova M.V., Panikovskaya T.Y., "Quality characteristics of conservative models of electric power systems," Messenger USTU, № 2 (10), 2000. Yekaterinburg: Ural State Technical University, 2000.

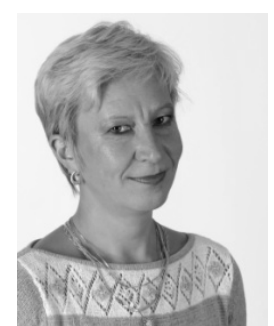

Tatiana Y. Panikovskaya, born in 1964 , graduated from Ural Polytechnic Institute named S.M. Kirova in 1986, received the qualification of electrical engineer, specialty "Electric systems and networks", in 1998 - that of Economist, specialty "Economics and Management of enterprises". Received the degree of Candidate of Technical Sciences in 1993, Assistant Professor since 1996.

Currently is working in the Automated Electric Systems Department and the Economy of Power and Marketing Department of Ural Federal University.

Her research interests include analysis for steady state EPS, operation and economics of electrical energy systems, restructuring of electric systems, and the development of algorithms for new electric markets. 